\title{
LA UTOPIAA Y SU POSIBILIDAD EN EL "PRINCIPIO ESPERANZA" DE ERNST BLOCH
}

\author{
Por: John Larry Rojas Castillo
}

\section{Resumen}

Este artículo busca comprender el sentido de la Utopía y su posibilidad en el primer tomo del principio Esperanza de Ernst Bloch. Se realiza una presentación básica de algunos elementos propios al filosofar del autor que permiten hacer inteligible la utopía como condición de la construcción de la humanidad a través del trabajo. De esta manera se concluye que la utopía es una forma humana y brillante de fantasía que se crea de forma sensata y esperanzada, que nace de la inconformidad con lo dado y que hace posible la crítica de lo presente, lo cual moviliza a la sociedad a construir un mundo sin clases en la que el hombre se naturalice y la naturaleza se humanice, es decir, en donde no exista alienación humana ni natural, sino armónico encuentro. De esta forma, la utopía se acompaña por la conciencia de lo imposible de una satisfacción plena de este deseo, pero al tiempo de lo fundamental de su búsqueda.

\footnotetext{
Abstract

The article aims to understand the essence of the utopian and its occurrence in the first volume of The Principle of Hope by Ernst Bloch. A basic introduction is given to the method the author philosophizes, which allows the utopian as an intelligible condition of the construction of humanity in the course of its work. In this way one can conclude that the utopian is a human form and a radiant fantasy that is formed in a hopeful and sensible manner. It also arises from the nonconformist way and makes it possible to criticize the present, which in turn the society congregates to construct a world without classes where man can naturalize and nature can humanize. In other words where neither human nor natural alienation exist, but only a harmonic encounter. It is essential that the utopian be complemented by the conscience of the impossible of a complete satisfaction of this wish, equal to the fundamental of its search
}

John Larry Rojas Castillo

Salvador Dali, La Pieta, 1982 
A Diana.
"El mundo se pone en movimiento Por el hambre y el amorn. Schiller

"Porque qué es saber de la vida, y tú Regalo de los dioses, fisonomía profética y del presentimiento, Voz encantada que me cantas."

Herder

Los acontecimientos históricos contemporáneos, junto con algunos importantes discursos de la época, permiten creer que el capitalismo se ha consolidado como la más perfecta manera de comprender y asumir la producción y lo humano, ya que como sistema mundo, abarca bajo sus condiciones todas las formas de vida y de relaciones sociales existentes.

Si bien esta consideración puede ser válida, son evidentes las contradicciones de este sistema. Ante esta posibilidad de la paradoja, de la perplejidad ante las falencias de este modo de producción y de relación humana, la filosofía de Ernst Bloch nos permite problematizar esta posición, ello por su comprensión del origen de lo humano, de la realidad y la historia.

Para nuestro autor la transformación, el cambio es el pathos fundante de lo real. Es por ello interesante acercarse a este filósofo con el fin de descubrir en él las características que nos permiten comprender la esperanza como impulso histórico permanente hacia la novedad.

En consecuencia, a lo largo de este escrito pretendo realizar una aproximación al tomo I del "Principio Esperanza" de Ernst Bloch, con el fin de exponer algunos elementos fundamentales que sustentan la posibilidad de la Utopía como dinámica viviente de lo real, como imagen que suscita la inconformidad frente al estado actual de cosas, impulsando así la aparición de lo Novum. Sin embargo, este Novum no está necesariamente determinado, sino que su realización efectiva depende del trabajo humano sobre la naturaleza.

Con el fin de recorrer esta cuestión desarrollaré tres movimientos teóricos básicos. En un primer momento, determinaré algunos elementos que permitan esclarecer en qué sentido Bloch es un filósofo marxista. Ellos son la comprensión de la realidad como materia en devenir siempre abierto y, por otro lado, la comprensión del hombre como sujeto transformador de lo real.

En un segundo momento, pretendo establecer cómo el autor presenta a la esperanza como el deseo que origina no solamente al hombre, sino al todo de lo real.

Finalmente, se mostrará cómo la fantasía utópica es posible a partir de la misma realidad material, pues se trata del impulso que nos permite representarnos un estado mejor de cosas que suscita la perplejidad, la crítica ante lo dado, generando así la posibilidad de la aparición de lo Novum. 


\section{Bloch y su Comprensión del Marxismo El Filosofar la Realidad}

Para Ernst Bloch "pensar significa traspasarn ${ }^{1}$, esto es, comprender la dinámica viviente de lo real; dicho vivir real cultiva en lo pasado la eclosión de lo nuevo, del futuro. El todo es devenir, es proceso material en infinita transformación. Lo real no es solamente lo dado, pues esto es apenas la semilla que hace posible la eclosión de lo nuevo.

Es precisamente en este punto en el que Bloch asume de manera radical el pensamiento de Marx en dos aspectos centrales, uno es la intelección de la realidad como movimiento abierto al cambio, a la mutación. Lo cual implica la comprensión del filosofar como una praxis concreta que pretende la transformación del mundo. El otro aspecto central es su comprensión histórico-antropológica, ya que para Bloch, siguiendo a Marx, el hombre es el responsable concreto, a través de su trabajo a lo largo del tiempo, de la realización de la utopía.

Con el fin de comprender la relación entre las filosofias de Bloch y Marx, es iluminador examinar el comentario que hace nuestro autor de las once tesis sobre Feuerbach. ${ }^{2}$

Para Bloch la undécima tesis muestra el profundo sentido que anima el filosofar

\footnotetext{
${ }^{1}$ BLOCH, Ernst, El Principio Esperanza, Trad. Felipe González Vicen, Ed. Aguijar, Tomo I, p. XIV. En este escrito todas las notas se referirán a esta traducción.

${ }^{2}$ Este trabajo es desarrollado de una manera muy cuidadosa en el Cap. 19 de] primer tomo de su obra. p.p. 243-283.
}

marxista. En la tesis referenciada, Marx no reprocha a los teóricos anteriores el no filosofar, sino no haber comprendido que la filosofía tiene como objetivo desencadenar una praxis concreta y transformadora del mundo. De esta manera, la reflexión criticada es la interpretación contemplativa, estática, lejana del mundo y, por ende, no comprometida con su transformación real concreta.

Es por ello que Bloch coloca como ejemplo del filosofar marxista el propio "Capital", en la medida en que esta obra es el logro del objetivo trazado por esta tesis: comprender, denunciar, a través de la razón, la contradicción implicada en la realidad humana propia del capitalismo, esto es, la alienación.

A partir de toda esta reflexión Bloch nos muestra cómo lo fundamental, lo propio de la filosofía marxista, está en su comprensión de la realidad como un hacerse, como un construirse a partir de la labor transformadora del hombre.

Así, la filosofía de Marx no se detiene en la comprensión arqueológica puramente contemplativa del pasado, es decir, no supone una actividad referida a lo ya plenamente definido e inmodificable; al contrario, ella pretende comprender lo vivido socialmente con el fin de revelar sus contradicciones y revolucionarias. Así, para Bloch:

La filosofía marxista es la filosofía del futuro, es decir, también del futuro en el pasado; en esta conciencia concentrada de frontera, la 
filosofía marxista es teoria-praxis de la tendencia inteligida, una teoría praxis viva, confiada en el acontecer, con la mirada fija en 10 novum. ${ }^{3}$

De esta manera, el filosofar intelige, para Bloch, la realidad como tendencia, como pathos de cambio, como impulso de transformación, a partir de lo cual comprendemos que el pasado implica la posibilidad real, siempre abierta de la novedad.

De esta forma, la realidad no es lo definido del pasado, sino que ella sólo se entiende en tanto horizonte abierto no para repetir lo hecho, sino para revolucionarlo, cultivando lo nuevo. La realidad es un hacer dinámico, es una tendencia viviente abierta al infinito horizonte histórico.

El hombre concreto en relaciones sociales, con sus necesidades y sus medios de satisfacción, es la profunda beta de la historia. Pero la historia, como se ha dicho, no es un proceso mecánico previsible, definido, sino el horizonte en el que se hace necesaria la posibilidad de la novedad cualitativa, es decir, donde lo Novum siempre es posible.

\section{El Hombre: centro de la preocupación del marxismo de Bloch ${ }^{4}$}

Para el autor, como se ha hecho evidente, la reflexión sobre el hombre es fundamental,

${ }^{3}$ Ibidem, p. XVIII

${ }^{4}$ En este momento se examinará el comentario de Bloch a las tesis IV, VI, VQ IX y X. Cfr. Ibidem. Pp 243-283. pues es él quien de manera concreta construye la realidad mediante su trabajo. Sin embargo, el capitalismo determina una serie de relaciones humanas de alienación del hombre respecto a la naturaleza. En este sentido, la pretensión de la utopía es la eliminación de esta relación negativa entre hombre y realidad natural.

En un primer momento, es claro que para Marx Feuerbach había realizado una comprensión profunda del concepto de alienación en Hegel y lo había aplicado en su crítica de la religión, develando que en ella el hombre proyecta, en un más allá irreal, sus necesidades concretas. Esta lectura llevó a Marx al descubrimiento de que es la relación entre los hombres el principio de toda verdadera teoría.

A su vez, el trabajo de Marx en la Gaceta Renana le permitió comprender que son las relaciones sociales concretas entre los hombres el origen general de la alienación religiosa y estatal, lo cual implica una ampliación profunda de lo realizado por Feuerbach.

En este sentido, Marx crítica a su maestro en tanto que si bien entiende que el hombre material es la clave para la comprensión de la realidad, ese hombre aparece como una entidad abstracta, pues se ignoran las relaciones sociales, concretas y múltiples en las que realmente aparece lo humano. Feuerbach se queda corto, pues no se percató de que la religión es apenas una manifestación, entre muchas otras, de la profunda aliena- 
ción humana nacida de las relaciones de clase y del trabajo. ${ }^{5}$

En consecuencia, Marx comprende al hombre, como ya se ha dicho, como un conjunto completo de relaciones sociales que determinan de manera concreta su libertad o enajenación. Así, la posibilidad de utopía o de condenación están, para el autor, en manos humanas en tanto conjunto social transformador de la naturaleza.

Por ello, Marx se propone revelar y denunciar las condiciones sociales en las cuales el sujeto pierde su realidad, ello con el fin de comprenderlas y transformarlas a partir de sí mismas. Para Bloch este es el objetivo concreto del materialismo histórico-dialéctico como comprensión verdadera del mundo.

En este sentido, se trata de abandonar la visión moderna que concibe la relación entre hombres como limitada a un vínculo netamente natural, para así cultivar un humanismo comunista. Bajo la pluma de Bloch:

Precisamente por ello el nuevo punto de vista, el punto de vista proletario, suprime tan poco el concepto axiológico de humanismo, que es él el que le hace prácticamente retornar a su propio lugar; y cuanto más científico es el socialismo, tanto más concretamente sitúa en el centro la preocupación

${ }^{5}$ Como ya habiamos dicho, Feuerbach nabía develado la alienación humana en la religión, la cual consiste en que el hombre material proyecta en un más allá la satisfacción de sus necesidades y carencias, de forma que se engaña a sí n1isn1o Por medio de las imágenes divinas que construye. por el hombre, y en la meta la superación real de su autoalienación ${ }^{6}$

En conclusión, podemos establecer que la intención de Bloch es realizar una reflexión filosófica marxista que comprenda la realidad como un impulso material en permanente devenir. Este filosofar es la develación de la realidad como totalidad que se impulsa permanentemente hacia lo nuevo.

Por otro lado, esta reflexión de Bloch es fruto de la lectura cuidadosa de la obra de Marx, en la cual se descubre el vínculo radical entre teoría y praxis, esto quiere decir, que la reflexión filosófica tiene su sentido en tanto devele el tejido concreto de lo humano implicado en las relaciones sociales, denunciando la alienación con el fin de transformarla en pos de la utopía.

Finalmente, en la lectura de los escritos juveniles de Marx, Bloch encuentra que el hombre es el centro de interés del verdadero pensar marxista, pues es el constructor concreto de la historia y, por ello, de la misma realidad.

\section{La esperanza como impulsora y origen de la realidad \\ Materia, impulso y deseo.}

Una vez realizada esta reflexión alrededor de las claves que nos permiten entender los

${ }^{6}$ Ibidem, p. 259. 
rasgos del filosofar utópico de Bloch, es necesario adentrarnos en la cosa misma, es decir, en la esperanza como impulso fundante de lo humano y de la realidad toda. Se trata de una comprensión profunda de las pasiones humanas a partir de sí mismas, comprendiendo su vitalidad, sus fuerzas de apetito o rechazo.

En un primer momento, vivir es, para el hombre, encontrarse atravesado por impulsos; es la conciencia del vacío, de la ausencia, de la negación que reclama un objeto externo, un otro más allá de sí mismo.

El vivir es búsqueda de lo no presente, dicha insatisfacción se impone como impulso, que palpita desde las profundidades íntimas de lo humano en tanto material.

Así, el cuerpo es el lugar material a partir del cual aparece para el hombre una compleja contradicción de impulsos vivientes. En consecuencia, la condición más fundante de lo humano es puro anhelar que palpita en su materialidad.

Como ya hemos dicho, este impulso se dirige a un objeto externo que determina la fuerza del anhelo, pues una vez alcanzado dicho objeto, entonces, el impulso desaparece. El animal y el hombre, en tanto lugares en los que aparece el vivir son, de manera inmediata, apetencia. Sin embargo, el impulso humano posee la particularidad de representar una imagen que define su fuerza de manera específica. Esto quiere decir que el ser huma- no no sólo padece la ausencia de lo apetecido, sino que al sufrir este anhelo organiza una serie de ideas a través de las cuales se realizan acciones. De esta manera, el animal sólo se satisface en lo buscado, mientras que el hombre además se lo imagina. ${ }^{7}$ El deseo es entonces la pasión específicamente humana en la cual el hombre es capaz de representar un estado de cosas anheladas que son el thelos de su acción.

Sin embargo, para el autor, es necesario distinguir entre desear y querer, ello en la medida en que el querer es una actividad concreta a través de la cual se busca la satisfacción del impulso, mientras que el deseo es un anhelar indeciso definido por la representación de ideas que no necesariamente son queridas. Lo deseado puede ser o no ejecutado, mientras que lo querido es lo que activamente se busca y construye.

Esto implica que el querer humano solamente nace a partir de los deseos, pues ellos delinean los pasos concretos que permiten al querer orientarse y encontrar un sentido concreto. De esta manera, el deseo es la partitura, el proceso ideal que guía el impulso en su satisfacción material.

El querer, al llenarse de sentido y color, al orientarse por la imagen representada de lo mejor, toma una fuerza cualitativamente humana. Es así como los hombres desean un estado más perfecto de cosas, lo cual pese a

${ }^{7}$ Cfr. Ibidem, p.30 
no ser satisfecho en lo real, sí les permite no adaptarse de manera vacía a lo dado.

En consecuencia, la satisfacción inmediata de las condiciones básicas de vida posibilita saciar un apetito; pero es propio del hombre no poder eliminar su deseo, en tanto que la situación ideal representada por los sujetos trasciende siempre las condiciones materiales actuales. En palabras del filósofo alemán:

"La joven que quisiera sentirse brillante y cortejada, el hombre que sueña con hazañas futuras llevan consigo la pobreza o la mediocridad como ropaje provisional. Ello no hace que el ropaje caiga, pero si que el hombre se adapte menos fácilmente a él. La mera apetencia y sus impulsos se contentan con lo que tienen, pero el deseo imaginativo en ellos tiende a más. ${ }^{8}$

De esta manera, podemos afirmar que son los deseos los que mueven a los hombres a lo largo de la historia, en tanto que ellos implican la representación de un estado de cosas mejor, el cual jamás es plenamente alcanzado a través de la pura e inmediata materialidad. La imaginación humana, la capacidad de soñar es, entonces, el rasgo propio del impulso viviente en el hombre.

\section{Del hambre a la esperanza}

Ahora bien, en un segundo momento es necesario mostrar cuál es la génesis de la

${ }^{8}$ Ibidem, p.31. esperanza en tanto deseo que expresa cualitativamente lo humano.

Entre los impulsos propios del hombre el hambre nos devela, de la forma más radical, la condición humana. Ello es claro en la medida en que no podemos subsistir sin satisfacer este apetito, si bien otros impulsos, dominación o amor, pueden ser sobrellevables en la vida. En este sentido es claro que debemos tratar de comprender, desde sí misma, esta necesidad que está en el origen de lo humano.

Así, el impulso fundamental humano es el hambre, el cual se transforma y define a través de la historia en sus condiciones concretas; pues todas las necesidades son determinadas socialmente tanto en su manifestación como en su satisfacción.

Del hambre proceden los impulsos en tanto tendencias, en tanto intenciones, en tanto afectos de apetencia o repugnancia. Estos afectos forman en el interior de lo humano un torrente pasional que el autor compara con el fluido sanguíneo y que en un primer momento, es netamente interno al hombre. Para el autor en esto consiste el estado de ánimo. El estado de ánimo es, en tanto íntimo, un fluir intransitivo que, en un segundo momento, toma cuerpo a través de las representaciones y las sensaciones. Como ya hemos dicho, las pasiones, en tanto afectos concentrados en una dirección, son tendencias hacia un algo, bien sea un objeto o una situación, ya sea concreta o imaginada. ${ }^{9}$ 
Ante esto el autor afirma, de la mano de Kierkegaard, que el estado de ánimo es lo propiamente existencial, es una condición originaria. Por lo cual se debe comprender en sí mismo, pues nos muestra la naturaleza inacabada del hombre, su capacidad de transformarse de manera siempre renovada. El ser humano busca de manera permanente alcanzar la satisfacción de sus necesidades, lo cual implica la metamorfosis permanente de sí mismo a través de la historia.

Por ello, para Bloch los afectos se dividen en afectos saturados y afectos de la espera. Los llamados saturados como la envidia, la avaricia y el respeto son impulsos referidos a objetos o situaciones accesibles en el mundo inmediato del sujeto; mientras que los afectos de la espera como el temor y la esperanza impulsan al hombre a través de lo dado a un estado futuro de cosas y de relaciones, en el que se puede encontrar mejor, de manera más plena y humana.

Ahora, si bien todos los afectos abren intencionalmente al hombre hacia el futuro, es decir, hacia un horizonte de posibilidades, los afectos de la espera realizan una apertura radical de dicho horizonte en tanto que implican los anhelos fundamentales, mientras que los afectos saturados sólo se refieren a un tiempo futuro en el que se puedan satisfacer necesidades precisas que no alejan

\footnotetext{
${ }^{9}$ En este sentido los afectos son intencionales, pero no podemos entender aqui un proceso en el que se distingue el contenido del acto intencional, a la manera de Husserl. Para Bloch los afectos son tendencias intencionales que tienen sentido com )Jeto por sí mismas, por su realidad anticipadora de lo deseado.
}

al sujeto de su inmediatez, de su estado actual de vida.

Entre los afectos de la espera, la esperanza es la pasión fundamental en la medida en que, como ya habíamos dicho, realiza en el hombre la apertura radical, la anticipación concreta de un mundo mejor y es, a la vez, el impulso por su formación y realización.

El hambre es la raíz de la cual crece la esperanza, ello en la medida en que la primera es una insaciable tendencia por llenar de manera real las necesidades humanas de libertad. Esto implica, necesariamente, que la no satisfacción actual aviva la esperanza futura. La esperanza implica la posibilidad específicamente humana de imaginar un estado mejor de cosas, lo cual da sentido al querer, pues dirige sus pasiones por encima del temor.

Ante esto, se hace necesario determinar las características de la nombrada fantasía utópica, ello con el fin de entender cómo ella hace posible la transformación permanente de un mundo mejor.

\section{La fantasía Utópica y su posibilidad real}

Los sueños diurnos aparecen como una fantasía que nace a partir de la constatación de que la realidad no está determinada de una manera necesaria, sino que depende, fundamentalmente, de los proyectos humanos que se construyen a través del trabajo. 
Estos sueños aparecen primeramente como la efervescencia de deseos e impulsos íntimos, que palpitan en el presente, en lo llegado a ser; esto abre al hombre a la fantasía de una situación humana mejor. Esta efervescencia es el primer correlato de la fantasía. En la medida en que, como se ha dicho, la realidad es proceso, es devenir de lo pasado hacia el porvenir, es decir, es futuro posible. Ahora, lo realmente posible es todo aquello cuyas condiciones no están todas presentes en el objeto mismo, ya sea porque no han madurado plenamente o porque es necesario que intervenga una nueva condición real.

Recordemos que, para el autor marxista, la realidad es ser material dialéctico, el cual es fundamentalmente inconcluso, abierto. Por ello la utopía no se puede negar de manera dogmática. En este orden de ideas, la validez del sueño diurno sólo puede evaluarse en las condiciones concretas en las que podría hacerse real. De esta manera, el segundo correlato de la fantasía utópica es lo realmente posible de una novedad en mediación creciente, esto es, las condiciones dialécticomateriales que de manera concreta realizan la utopía. Así, ella tiene correspondencia en la realidad como proceso, como devenir de lo Novum en la mediación concreta. Lo nuevo es entonces el fruto de un hacer que se hace concreto a partir de las condiciones del presente.

Para Bloch esta fantasía es realmente construida a través del trabajo del hombre, el cual se diferencia del consumir animal porque la inteligencia humana proyecta sobre la naturaleza el objetivo final de su actividad; así el ser humano no solamente imprime a los objetos su forma, sino que en el resultado trabajado se encuentra materializado su proyecto, su fantasía. De esta manera la esperanza lleva al hombre real a soñar un proyecto que anhela y construye a través de su trabajo efectivo sobre el mundo.

El sueño diurno consiste, en consecuencia, en el proyecto en el cual se vislumbra un mundo humano en el que no exista opresión. El motor de este sueño es la esperanza y su realización es garantizada por el trabajo. Es preciso enfatizar aquí lo diurno del sueño, pues para el autor no se trata de una evasión de la situación dada en un espiritual más allá, sino de un proyecto que parte del estado de vigilia, de consciencia de lo real y que, por ello, impulsa al hombre en la realización material de una vida propiamente humana. ${ }^{10}$

Esta reflexión sobre las condiciones de realización de la utopía concreta y acerca de las limitación de diversas filosofías que no comprenden adecuadamente la realidad dialéctico-material, llevan al autor a investigar de manera detallada los conceptos de Novum y Ultimum; ello con el fin de comprender el

\footnotetext{
${ }^{10}$ Ante esta consideración, el autor crítica a] positivismo en tanto que éste considera que la historia no posee un proceso, un movimiento. Además, crítica cierta visión marxista que, si bien concibe un devenir histórico, se queda en suponerlo como una sucesión de acontecimientos y épocas como totalidades conclusas, sin comunicación entre sí, lo cual elimina la comprensión dialéctica del proceso. Cfr. Ibidem, p.p. 187-189.
} 
sentido y la sustentación del optimismo militante que implica la esperanza.

\section{Lo Novum, lo Ultimum y lo Primum}

Tal y como se ha determinado, lo real es movimiento dialéctico material que tiende a un Ultimum. Para Bloch este Ultimum es la utopía en la que se suprime la opresión entre los hombres y se da una relación humanizada con la naturaleza; para el autor este estado, que siempre está por hacerse, implica una actitud Ilamada optimismo militante. Este optimismo lleva a los proletarios a construir de manera concreta la utopía y, por ello, supone necesariamente la exposición teórica de las condiciones que hacen posible la mencionada realización.

Contrariamente a esta propuesta, para el pensamiento cristiano la realidad ha sido creada de la nada -ex nihilo- por un principio supremo que teleológicamente, gobierna y dirige hacia él la naturaleza y la historia. Este creador es principio y fin, es decir, de él y hacia él deviene la realidad en la medida en que él es su fuente y origen.

De esta manera la noción de Ultimum en el cristianismo, y en las filosofías que beben de su fuente, deja de lado la noción de lo Novum: Ello porque Dios ha determinado necesariamente todo el proceso material e histórico, pues Él mismo es el principio de la realidad. ${ }^{11}$
Para Bloch la capacidad materialista dialéctica de la historia está en que siempre abre el horizonte al Novum, el cual no es de ninguna manera determinado lógicamente por el Primum, sino que más bien ese origen es aniquilado de manera permanente por la novedad. Esa novedad consiste en la aparición de lo posible real lo cual es, como ya hemos expuesto, siempre abierto y depende de manera concreta del trabajo de los hombres. Lo nuevo no es la oposición simple a la repetición mecánica, como tampoco la proyección de lo pasado en un tiempo actual, sino que es un aparecer, un manifestarse de lo real en el horizonte indeterminado de la historia. ${ }^{12}$

Lo real como movimiento dialéctico debe entenderse como proceso o devenir que pretende realizar la utopía concreta como un camino en el que existe lo Novum, ello es un tipo de repetición específica que consiste en la revelación, en la denuncia de que cada momento de la historia no es pleno, sino que implica una tendencia hacia un contenido real no alcanzado.

Ese contenido real es el que siempre aparece como Ultimum en tanto supresión de lo Novum, como plena realización de la uto-

\footnotetext{
${ }^{11}$ En coherencia con esto, Hegel concibe la realidad desde un punto de vista netamente idealista, como mediación o ser para si de un ser primigenio en si, de forma que Primum y realizaciónmediación son una y la misma cosa. Recordemos también las teorias agustinianas del devenir de la historia hacia el dia del descanso en Dios y de las especies seminales en la Ciudad de Dios.

${ }^{12}$ En este momento el autor crítica la visión mecánica y repetitiva de lo nuevo en Bergson, asi como la equiparación entre 10 nuevo y la moda. Cfr. BLOCI-I, Ernst, El Principio Esperanza, Trad. Fe1ipe González Vicen, Ed. Agui1ar, Tomo 1, p. 194.
} 
pía. ${ }^{13}$ Así, lo Ultimum no es un objeto determinado estático o rígido, sino el objetivo de la pasión, de la aspiración humana por un mundo de libertad y de plena reconciliación con la naturaleza. Por ello, dicho objetivo es el horizonte de lo humano ante cuya visión nos sentimos interpelados, insatisfechos; ello origina entonces una actitud crítica frente a lo dado y, por ello, genera la motivación que lleva al hombre a una actividad siempre creadora.

\section{La Posibilidad objetivamente real, actuali- dad de la fantasía Utópica.}

Finalmente, se hace necesario preguntar por qué existe la posibilidad en la misma realidad, es decir, por qué razones la realidad misma es posibilidad. ${ }^{14}$

Para nuestro autor en el materialismo-histórico-dialéctico la materia implica, por un lado, la posibilidad, el ente-que todavía-no-es y, por otro, el principio que pone en acto esta infinita fecundidad. Esta concepción necesariamente abre al horizonte de la historia en la que esta materia se realiza Wirklichkeit a sí misma. Bajo la pluma de Bloch: "la materia es la posibilidad real para todas las formas que se hallan latentes en su seno y se desprenden de ella por medio del proceso". ${ }^{15}$

\footnotetext{
${ }^{13}$ Cfr. Ibidem., p.195.

${ }^{14}$ Para desarrollar esta cuestión es necesario determinar los elementos clave de la ontologia del materialismo de la esperanza, lo cual sólo se logra a través de una interesante discusión con la tradición filosófica, la cual; dada su complejidad especulativa, no vamos a tratar en este momento.

${ }^{15}$ Ibidem, p. 229.
}

La realidad así concebida contiene en sí no un proceso mecánico cerrado, sino un devenir dialéctico que conduce necesariamente a la búsqueda progresiva de la libertad a lo largo de la historia. Así, en palabras de Bloch "Sin materia no es aprehensible ningún suelo de la anticipación -real-; sin anticipación -real- no es aprehensible ningún horizonte de la materia» ${ }^{16}$.

Ahora bien, la materia no está definida de manera absoluta, como si se tratara de semillas que después simplemente se revelan, sino que el germen de lo real es desarrollado plenamente, en sus condiciones concretas, a través del trabajo humano. Esto quiere decir, que lo real se desenvuelve a través del hombre trabajador que realiza de manera novedosa la utopía buscada. Lo real es posibilidad, es potencia de ser y actividad concreta que lo realiza.

Lo posible, entonces, es lo propio a la cosa de acuerdo con su estructura, esto es, de acuerdo con las condiciones internas y externas que definen su ser; estas condiciones se entrecruzan mutuamente de manera compleja. Así, para seguir con la misma analogía, el fruto desarrollado plenamente en el interior de la planta, puede no manifestarse por la falta de las condiciones externas adecuadas. En palabras de Bloch: "Posibilidad significa aquí tanto poder interno, activo, como poderser-hecho externo, pasivo." ${ }^{17}$

\footnotetext{
${ }^{16}$ Ibidem, p. 231

${ }^{17}$ Ibidem, p. 226.
} 
En la sociedad, las condiciones de aparición de un nuevo momento en su historia implican también esta compleja red que se trama de manera necesaria entre sujetos y situación social general.

De esta manera, las condiciones internas o el poder-hacer-algo-distinto se entrecruzan con las condiciones externas o el poder-hacersedistinto, de forma que los hombres poseen en sus manos, en su transformación del mundo, la posibilidad de salvación, esto es, de cultivo de las condiciones estructurales de su propia realidad de manera que florezca la utopía de la libertad.

Sin embargo, el florecimiento de lo Novum implica un azar mediado ${ }^{18}$; ello en la medida en que los hombres construyen por sí mismos, de forma a veces no consciente, su propia realidad; entonces, en la inseguridad de las situaciones se implica también la posibilidad de la eclosión, a partir de las condiciones construidas, de la condenación. Al ser la utopía el sueño de hombres finitos, su realización es frágil, pues la misma posibilidad de su realidad implica, igualmente, la potencia de la frustración.

Pero esta paradoja nos devela la fecundidad de lo posible, nos muestra que el horizonte de lo que podemos hacer de nuestra vida es mucho más amplío que el recuerdo de lo que hemos sido. De esta manera el temor y la

${ }^{18}$ Esto implica que lo azaroso depende de las condiciones de desarrollo que median lo posible real en la cosa, no se trata, entonces, de un azar caprichoso, sino de lo contingente inherente al poder aparecer de lo nuevo. esperanza se mezclan en la humanidad de manera inevitable.

Es muy sugerente reflexionar un poco sobre cómo Bloch, exiliado en los Estados Unidos, pensó estas ideas en el ambiente de la guerra, pues su filosofar es un gran esfuerzo por soñar la humanidad sobre los escombros de su época. El autor muestra que la fantasía que se crea con los ojos abiertos y centrados en lo que ahora existe, es la única posibilidad de inconformidad, de crítica y de activo construir de una sociedad sin clases en la que el hombre se naturalice y la naturaleza se humanice, es decir, en donde no exista alienación humana ni natural, sino armónico encuentro. Ello con la conciencia de lo imposible de una satisfacción plena de este deseo, pero de lo fundamental de su búsqueda.

De esta manera, es necesario cultivar los sueños diurnos en tanto expresiones de una esperanza activa que transforma lo real, ejecutando así nuevas mediaciones que permitan acercar más lo actual a la utopía.

Sólo en el sueño diurno y la actividad valiente y transformadora, se abre el horizonte futuro por encima del miedo y se cultivan las condiciones que hacen devenir la utopía de lo posible, a lo real. 